\title{
Influence of Magnetic Moment Formation on the Conductance of Coupled Quantum Wires
}

\author{
V. I. Puller \\ Department of Physics, Queens College, \\ the City University of New York, Flushing, New York 11367, USA \\ L. G. Mourokh \\ Department of Physics and Engineering Physics, \\ Stevens Institute of Technology, Hoboken, New Jersey 07030, USA \\ J. P. Bird \\ Department of Electrical Engineering, University at Buffalo, \\ the State University of New York, Buffalo, NY 14260-1920, USA \\ Y. Ochiai \\ Department of Electronics and Mechanical Engineering, 1-33, \\ Yayoi-cho, Inage-ku, Chiba City, Chiba, 263-8522 Japan
}




\begin{abstract}
In this report, we develop a model for the resonant interaction between a pair of coupled quantum wires, under conditions where self-consistent effects lead to the formation of a local magnetic moment in one of the wires. Our analysis is motivated by the experimental results of Morimoto et al. [Appl. Phys. Lett. 82, 3952 (2003)], who showed that the conductance of one of the quantum wires exhibits a resonant peak at low temperatures, whenever the other wire is swept into the regime where local-moment formation is expected. In order to account for these observations, we develop a theoretical model for the inter-wire interaction that calculated the transmission properties of one (the fixed) wire when the device potential is modified by the presence of an extra scattering term, arising from the presence of the local moment in the swept wire. To determine the transmission coefficients in this system, we derive equations describing the dynamics of electrons in the swept and fixed wires of the coupled-wire geometry. Our analysis clearly shows that the observation of a resonant peak in the conductance of the fixed wire is correlated to the appearance of additional structure (near 0.75 or $0.25 \cdot 2 e^{2} / h$ ) in the conductance of the swept wire, in agreement with the experimental results of Morimoto et al.
\end{abstract}




\section{INTRODUCTION}

The low-temperature conductance of quantum point contacts (QPCs) is well known to be quantized in units of $2 e^{2} / h$, a phenomenon that can be explained in terms of a simple transmission (Landauer) picture in which the influence of electron-electron interactions is neglected [1]. While this model is remarkably successful in accounting for the observation of conductance steps at integer units of $2 e^{2} / h$, it is unable to explain the origin of the additional conductance plateau, observed near $0.7 \cdot 2 e^{2} / h$ in numerous experiments. (For an overview of this issue, see Ref. [2].) While many different theoretical models have been proposed to account for the origins of the 0.7 feature, there is a wide consensus that it is likely associated with some novel many-body effect. One of the most convincing explanations (although not yet commonly accepted) is the development of a net magnetic moment in the QPC when it is almost pinched off [3, 4, 5]. In our recent work [6, 7, 8] we provided experimental and theoretical support for this idea. The device structure that we have studied experimentally in Ref. [6] is shown in Fig. 1 and was formed in the two-dimensional electron gas of a GaAs/AlGaAs quantum well. The device was realized by means of electron-beam lithography, and lift-off of Ti-Au gates. These gates were formed on a Hall bar with eight ohmic contacts, positioned uniformly along its upper and lower edges. In suitable combinations, these contacts could be used to make four-probe measurements of the conductance of either wire, or of the quantum dot itself (as indicated in Fig. 1). Of particular interest here is the non-local measurement (right panel) that can be made by measuring the conductance through one (fixed) wire as the gate voltage $\left(V_{g}\right)$ applied to the other (swept) wire is varied. The key result of our experiment is that as the swept wire pinches off, a resonant enhancement of the conductance of the fixed wire is observed. A qualitative theoretical explanation of this phenomenon was given in Ref. 7]. Based on a modified Anderson Hamiltonian, we showed that the resonant interaction with the local magnetic moment formed in the swept wire leads to an additional positive contribution to the density of states of the fixed wire and, consequently, to an enhancement of its conductance. While this analysis provides a qualitative understanding of the resonant interaction between the quantum wires, the tunnel matrix elements involved in the Anderson Hamiltonian are generally unknown and have thus far been used as fitting parameters. In addition, the influence of the specific device geometry

on these matrix elements has thus far been neglected, even though geometry-related effects 
are known to be important for the description of scattering in one-dimensional structures 9, 10]. To overcome these shortcomings, in this paper we present a more comprehensive theory for the electron dynamics in the coupled-wire system of Fig. 1 and attempt to calculate the amplitude of the resonant inter-wire interaction without the assumption of the localized state formed in the swept wire. To this end, we further develop the approach introduced in our previous paper [8], where the conductance of a single quantum wire was determined. In this work, we obtained such features as an additional $0.75 \cdot 2 e^{2} / h$ plateau for ferromagnetic coupling between the local moment in the QPC and the conducting electrons and a $0.25 \cdot 2 e^{2} / h$ plateau for the antiferromagnetic coupling, in agreement to the research of Refs. [11, 12]. In the present paper we calculate the single-electron transmission properties of the fixed wire in a device potential that is modified by the presence of an extra scattering term, arising from the presence of a local magnetic moment in the swept wire. The formulation of this idea is given in Section II, where we derive equations describing the dynamics of electrons in the swept and fixed wires. In Section III, we determine the transmission coefficient and conductance for the fixed wire and compare these expressions to the results of Ref. 8]. In particular, we show that an additional peak in the conductance of the fixed wire is correlated to the appearance of additional plateaus (at $0.75 \cdot 2 e^{2} / h$ or $0.25 \cdot 2 e^{2} / h$ ) in the conductance of the swept wire in agreement with the experimental results of Ref. [6]. The conclusions are presented in Section IV.

\section{ELECTRON MODES IN THE COUPLED QUANTUM WIRE STRUCTURE}

We start our description of electron dynamics in the coupled quantum wire structure from the following single-particle Hamiltonian

$$
\hat{H}_{0}=K_{x}+K_{y}+U(x)+W(y)+V(x, y)-J(x, y) \hat{\vec{\sigma}} \cdot \hat{\vec{S}}
$$

where $K_{x}$ and $K_{y}$ are the kinetic energy operators for an electron localized in the 2D plane, $W(y)$ is the double-well potential describing the two quantum wires (Fig. 2, center panel), $V(x, y)$ is the potential of the tunnelling channel connecting the two wires (Fig. 2, right panel), and $U(x)$ describes the smooth bottleneck shape of the quantum wire channels. The last term simulates exchange coupling between the conductance electrons (Pauli

matrices $\hat{\vec{\sigma}}$ ) and the local moment, $\hat{\vec{S}}$, which is assumed to be a spin- $1 / 2$ magnetic mo- 
ment with $J(x, y)$ as the coordinate-dependent exchange coupling constant. The potentials $U(x), J(x, y)$, and $V(x, y)$ vanish as $x \rightarrow \pm \infty$. The potential $V(x, y)$ is very sharp in comparison with the variation of $U(x)$ in the $x$-direction due to the narrowness of the windows connecting the QPCs and the quantum-dot region. $J(x, y)$ has an $x$-dependence similar to that of $U(x)$, since the spatial characteristics of the local magnetic moment formed in the conducting channel are determined by the shape of this channel.

We write the Schrödinger equation in the form

$$
\hat{H}_{0} \hat{\psi}(x, y)=E \hat{\psi}(x, y)
$$

where the symbol "hat" in this and subsequent equations is used for operators and wave functions in the four-dimensional spin space of the two spins. The basis vectors in this space (uncoupled representation) are given by [13]

$$
\hat{\chi}_{1}=\left|\uparrow_{e}\right\rangle\left|\uparrow_{S}\right\rangle, \hat{\chi}_{2}=\left|\downarrow_{e}\right\rangle\left|\downarrow_{S}\right\rangle, \hat{\chi}_{3}=\left|\uparrow_{e}\right\rangle\left|\downarrow_{S}\right\rangle \text {, and } \hat{\chi}_{4}=\left|\downarrow_{e}\right\rangle\left|\uparrow_{S}\right\rangle \text {, }
$$

where $\left|\uparrow_{e}\right\rangle\left(\left|\downarrow_{e}\right\rangle\right)$ and $\left|\uparrow_{S}\right\rangle\left(\left|\downarrow_{S}\right\rangle\right)$ are spin-up (spin-down) states of the electron spin, $\vec{\sigma}$, and the local moment spin, $\vec{S}$, respectively. The canonical transformation to the coupled representation is discussed in Appendix A.

The solution of the Schrödinger equation, Eq. (2), can be expanded in terms of the spin functions, Eq. (3), as

$$
\hat{\psi}(x, y)=\sum_{\alpha=1}^{4} \hat{\chi}_{\alpha} \psi_{\alpha}(x, y) .
$$

Following the procedure of Ref. 9] we expand the full wave functions in terms of different propagating modes

$$
\hat{\psi}(x, y)=\sum_{n} \hat{\varphi}_{n}(x) \Phi_{n}(y)
$$

with the transverse structure of $n^{t h}$ mode given by the solutions of the equation

$$
\left[K_{y}+W(y)\right] \Phi_{n}(y)=E_{n} \Phi_{n}(y)
$$

Correspondingly, the wave functions $\hat{\varphi}_{n}(x)$ obey the coupled equations

$$
\left[E-E_{n}-K_{x}-U_{n}(x)\right] \hat{\varphi}_{n}(x)=\sum_{m \neq n}\left(V_{n m}(x)-J_{n m}(x) \hat{\vec{\sigma}} \cdot \hat{\vec{S}}\right) \hat{\varphi}_{m}(x)
$$

where 


$$
\begin{aligned}
V_{n m}(x) & =\int d y \Phi_{n}^{*}(y) V(x, y) \Phi_{m}(y), \\
J_{n m}(x) & =\int d y \Phi_{n}^{*}(y) J(x, y) \Phi_{m}(y),
\end{aligned}
$$

and $U_{n}(x)=U(x)+V_{n n}(x)$.

In the following analysis we make a number of simplifications in Eq. (77). First, we note that if the wires are well separated, the wave functions $\Phi_{n}(y)$ are strongly localized in one of the two wires, allowing us to distinguish the modes propagating in each of the wires. We assume that the shape of the confining potential $W(y)$ is such that one of the wires is close to pinch off (the swept wire), i.e. it has only one propagating mode (described by the wave function $\left.\hat{\varphi}_{0}(x)\right)$ with the transverse confinement (subband bottom) energy, $E_{0}$, less than the Fermi energy, whereas the other wire (the fixed wire) has several propagating modes (Figure 3). The localized magnetic moment is supposed to form in the only subband of the swept wire, hence the exchange coupling can be approximated as $J_{n m}(x)=\delta_{n, 0} \delta_{m, 0} J(x)$. Thus the system of equations is reduced to

$$
\left[E-E_{0}-K_{x}-U_{0}(x)+J(x) \hat{\vec{\sigma}} \cdot \hat{\vec{S}}\right] \hat{\varphi}_{0}(x)=\sum_{n \geq 1} V_{0 n}(x) \hat{\varphi}_{n}(x)
$$

and

$$
\left[E-E_{n}-K_{x}-U_{n}(x)\right] \hat{\varphi}_{n}(x)=\sum_{m} V_{n m}(x) \hat{\varphi}_{m}(x) \text { for } n \geq 1 .
$$

Furthermore, relying on the large energy separation between the subbands, in comparison with the magnitudes of $V_{n m}(x)$ and $J(x)$, we will neglect interaction between different subbands of the fixed wire, effectively restricting our analysis to a two-subband model, i.e. studying the only subband of the swept wire and $n^{t h}$ subband of the fixed wire. The coupled equations for this pair of subbands are

$$
\begin{gathered}
{\left[E-E_{0}-K_{x}-U_{0}(x)+J(x) \hat{\vec{\sigma}} \cdot \hat{\vec{S}}\right] \hat{\varphi}_{0}(x)=V_{n}(x) \hat{\varphi}_{n}(x),} \\
{\left[E-E_{n}-K_{x}-U_{n}(x)\right] \hat{\varphi}_{n}(x)=V_{n}(x) \hat{\varphi}_{0}(x),}
\end{gathered}
$$

where we have introduced $V_{n}(x)=V_{0 n}(x)=V_{n 0}(x)$.

Eqs. $(12,13)$ can be decoupled using Green's functions:

$$
\hat{G}_{0}(\epsilon)=\left[\epsilon-K_{x}-U_{0}(x)+J(x) \hat{\vec{\sigma}} \cdot \hat{\vec{S}}\right]^{-1}
$$


and

$$
\hat{G}_{n}(\epsilon)=\left[\epsilon-K_{x}-U_{n}(x)\right]^{-1} .
$$

With these Green's functions Eqs. $(12,13)$ can be formally integrated as

$$
\hat{\varphi}_{0}(x)=\hat{G}_{0}\left(E-E_{0}\right) V(x) \hat{\varphi}_{n}(x)
$$

and

$$
\hat{\varphi}_{n}(x)=\hat{G}_{n}\left(E-E_{n}\right) V(x) \hat{\varphi}_{0}(x) .
$$

Accordingly, we obtain

$$
\left[E-E_{0}-K_{x}-U_{0}(x)+J(x) \hat{\vec{\sigma}} \cdot \hat{\vec{S}}\right] \hat{\varphi}_{0}(x)=V(x) \hat{G}_{n}\left(E-E_{n}\right) V(x) \hat{\varphi}_{0}(x),
$$

and

$$
\left[E-E_{n}-K_{x}-U_{n}(x)\right] \hat{\varphi}_{n}(x)=V(x) \hat{G}_{0}\left(E-E_{0}\right) V(x) \hat{\varphi}_{n}(x) .
$$

The Green's function $\hat{G}_{n}(\epsilon)$ is a scalar Green's function, i.e. it is a unit matrix in the uncoupled spin space, whereas $\hat{G}_{0}(\epsilon)$ has a more complicated structure. Nevertheless, it can be expressed in terms of two scalar Green's functions (see the derivation in Appendix B) as

$$
\hat{G}_{0}(\epsilon)=\frac{1}{4}\left[3 g^{t}(\epsilon)+g^{s}(\epsilon)\right] \hat{I}+\frac{1}{4}\left[g^{t}(\epsilon)-g^{s}(\epsilon)\right] \hat{\vec{\sigma}} \cdot \hat{\vec{S}},
$$

where

$$
g^{t}(\epsilon)=\left[\epsilon-K_{x}-U(x)+J(x)\right]^{-1}
$$

and

$$
g^{s}(\epsilon)=\left[\epsilon-K_{x}-U(x)-3 J(x)\right]^{-1} .
$$

Now we are able to redefine the scalar potentials, as

$$
\tilde{U}_{0}(x, E)=U_{0}(x)+V(x) \hat{G}_{n}\left(E-E_{n}\right) V(x)
$$

and

$$
\tilde{U}_{n}(x, E)=U_{n}(x)+v_{n}(x, E)=U_{n}(x)+V_{n}(x) \frac{1}{4}\left[3 g^{t}\left(E-E_{0}\right)+g^{s}\left(E-E_{0}\right)\right] V_{n}(x),
$$

and introduce the tunneling-induced exchange coupling of electrons in the fixed wire to the local magnetic moment,

$$
j_{n}(x, E)=-V_{n}(x) \frac{1}{4}\left[g^{t}\left(E-E_{0}\right)-g^{s}\left(E-E_{0}\right)\right] V_{n}(x) .
$$


As a result, we obtain the following equations for the description of electron dynamics in the swept and fixed wires in the form

$$
\left[E-E_{0}-K_{x}-\tilde{U}_{0}(x)+J(x) \hat{\vec{\sigma}} \cdot \hat{\vec{S}}\right] \hat{\varphi}_{0}(x)=0
$$

and

$$
\left[E-E_{n}-K_{x}-\tilde{U}_{n}(x)+j(x, E) \hat{\vec{\sigma}} \cdot \hat{\vec{S}}\right] \hat{\varphi}_{n}(x)=0 .
$$

Although the form of these two equations is very similar, and they can be both treated in the same manner (as is discussed in the next Section), the results they yield will differ, depending on the specific shapes of the potentials and exchange couplings. In particular, while the shape of the coupling $J(x)$ in Eq. (26) is smooth, similar to that of the potential $U(x)$, the exchange constant $j(x)$ of Eq. (27) is proportional to the potential $V(x)$, and therefore is sharper than the bottleneck potential $U(x)$.

\section{CALCULATIONS OF THE TRANSMISSION COEFFICIENT AND CON- DUCTANCE FOR THE SWEPT AND FIXED WIRES}

In our previous paper [8], we determined the transmission coefficient and the conductance of a single QPC, expanding functions $\tilde{U}_{0}(x)$ and $J(x)$ involved in Eq.(26) into series near their maxima (i.e. representing them as inverted parabolas) as

$$
\tilde{U}_{0}(x)=\tilde{U}_{0}(0)+\left.\frac{x^{2}}{2} \frac{\partial^{2} \tilde{U}_{0}(x)}{\partial x^{2}}\right|_{x=0}=\tilde{U}_{\max }-\frac{m \omega_{U}^{2} x^{2}}{2}
$$

and

$$
J(x)=J(0)+\left.\frac{x^{2}}{2} \frac{\partial^{2} J(x)}{\partial x^{2}}\right|_{x=0}=J_{\max }-\frac{m \omega_{J}^{2} x^{2}}{2} .
$$

The transmission coefficient for the inverse parabolic barrier $u(x)=-m \omega^{2} x^{2} / 2$ is given by [14]

$$
t(\eta)=\left[1+e^{-2 \pi \eta}\right]^{-1 / 2}
$$

where $\eta=\epsilon / \hbar \omega$, and the energy, $\epsilon$, is measured from the top of the barrier. Thus, the transmission coefficients of the swept wire can be written as

$$
T_{0 t}=t\left(\frac{\epsilon-\tilde{U}_{\max }+J_{\max }}{\hbar \omega_{-}}\right)
$$


and

$$
T_{0 s}=t\left(\frac{\epsilon-\tilde{U}_{\max }-3 J_{\max }}{\hbar \omega_{+}}\right),
$$

where $\omega_{-}=\sqrt{\omega_{U}^{2}-\omega_{J}^{2}}, \omega_{+}=\sqrt{\omega_{U}^{2}+3 \omega_{J}^{2}}$. Assuming the equivalence of all initial spin orientations, we obtain the conductance of the swept wire as

$$
\begin{array}{r}
G_{S W}=\frac{2 e^{2}}{h}\left[\frac{3}{4}\left|T_{0 t}\right|^{2}+\frac{1}{4}\left|T_{0 s}\right|^{2}\right]= \\
\frac{2 e^{2}}{h}\left[\frac{3}{4}\left|t\left(\frac{\epsilon-\tilde{U}_{\max }+J_{\max }}{\hbar \omega_{-}}\right)\right|^{2}+\frac{1}{4}\left|t\left(\frac{\epsilon-\tilde{U}_{\max }-3 J_{\max }}{\hbar \omega_{+}}\right)\right|^{2}\right] .
\end{array}
$$

The most important feature of the transmission coefficients is that the transmission probability, $|t(\eta)|^{2}$, is very close to a step function. This step-like structure causes the conductance to reproduce the step-like behavior of the 0.7-anomaly. In the case of ferromagnetic coupling between the electrons and local magnetic moment, $J_{\max }>0$, our model gives an additional conductance step at $0.75 \times 2 e^{2} / h$, as

$$
G_{S W}=\frac{2 e^{2}}{h} \begin{cases}0, & \text { if } \epsilon<\tilde{U}_{\max }-J_{\max } \\ 0.75, & \text { if } \tilde{U}_{\max }-J_{\max }<\epsilon<\tilde{U}_{\max }+3 J_{\max } \\ 1, & \text { if } \epsilon>\tilde{U}_{\max }+3 J_{\max }\end{cases}
$$

It is interesting to point out that for antiferromagnetic coupling, $J_{\max }<0$, we obtain a conductance step at $0.25 \times 2 e^{2} / h$, which has been observed in experiments [15] and densityfunctional simulations [4], as

$$
G_{S W}=\frac{2 e^{2}}{h} \begin{cases}0, & \text { if } \epsilon<\tilde{U}_{\max }-3\left|J_{\max }\right| \\ 0.25, & \text { if } U_{\max }-3\left|J_{\max }\right|<\epsilon<\tilde{U}_{\max }+\left|J_{\max }\right| \\ 1, & \text { if } \epsilon>\tilde{U}_{\max }+\left|J_{\max }\right|\end{cases}
$$

The idea that the 0.7-anomaly is caused by singlet-triplet splitting of the first plateau, into the triplet part contributing $3 / 4(=0.75)$ and the singlet part contributing $1 / 4(=0.25$, was suggested in Refs. 11] and [12]. However, these theories failed to reproduce the correct behavior of 0.7-anomaly with variations of temperature, concentration and source-drain bias. Correspondingly, the model used in the present paper is also too primitive but it can be improved, in particular, by using the results of density functional modelling [4, 5] to specify the shape and strength of the exchange coupling $J(x, y)$ by comparing phenomenological parameters to experimental data. It should be also noted that in experiments the actual 
position of the "0.7-plateau" varies between 0.5 and 0.8 for samples having different electron concentrations, gate voltages, and source-drain biases (see 2] and references therein) and, accordingly, the theoretical explanations providing the " 0.75 " result cannot be ruled out especially in a view of experimental observation of the 0.25-plateau [15].

The method of calculation of the fixed wire conductance is very similar to that of the swept wire. However, the exchange and scattering potentials, involved in Eq. (26) for the swept wire and in Eq. (27) for the fixed wire, are different, leading to the differences in the behavior of the conductance. One of the main differences is that the tunneling channel, whose width is characterized by the width of potential $V_{n}(x)$, is narrow in comparison to the extent of the bottleneck potential of a quantum wire, described by $U_{n}(x)$, and the corrections associated with this tunneling appear as a peak or a dip on top of the potential.

To evaluate Eq. (27), we rewrite it in the coupled representation (see appendix A, with the prime to be omitted below) as

$$
\left(E-E_{n}-K_{x}-U_{n}(x)-v_{n}(x)+j_{n}(x, E)\right) \varphi_{n \alpha}(x)=0
$$

for $\alpha=1,2,3$ and

$$
\left(E-E_{n}-K_{x}-U_{n}(x)-v_{n}(x)-3 j_{n}(x, E)\right) \varphi_{n 4}(x)=0
$$

for $\alpha=4$. The exchange-independent solutions can be found from the equation

$$
\left(E-E_{n}-K_{x}-U_{n}(x)-v_{n}(x)\right) \chi_{n k}^{ \pm}(x)=0,
$$

where $k=\frac{1}{\hbar} \sqrt{2 m\left(E-E_{n}\right)}$, and we denote the transmission coefficient associated with these solutions as $t_{n}\left(E-E_{n}\right)$.

We can express the exchange term, $j_{n}(x, E)$ in terms of the transmission coefficients of the swept wire. In this, we employ the approximation of inverse parabolicity of the barrier in the swept wire to the Green's functions involved in the definition of the exchange term,

$$
j_{n}(x)=-V_{n}(x) \frac{1}{4} \int d x^{\prime}\left[g^{t}\left(x, x^{\prime}, E-E_{0}\right)-g^{s}\left(x, x^{\prime}, E-E_{0}\right)\right] V_{n}\left(x^{\prime}\right) .
$$

Using the properties of the Green's functions of the inverse parabolic barrier (see Appendix $\mathrm{C})$, we find that the energy dependence of the exchange term is determined by the difference of the transmission coefficients as

$$
j_{n}(x, E) \sim\left[T_{0 t}\left(E-E_{0}\right)-T_{0 s}\left(E-E_{0}\right)\right] j_{n}(x) .
$$


The contributions of the exchange interaction have different signs for the singlet and triplet states and appear as a peak and a dip, respectively, on top of the bottleneck potential $\tilde{U}(x)=U(x)+v(x)$ (see Figure 4). The dip leads to the occurrence of localized states inside the potential of the fixed wire modifying its conductive properties. We consider two possible situations.

1. Ferromagnetic coupling $\left(j_{n}(x, E)>0\right)$.

In this case the triplet states experience a dip in the potential, and the energy of the quasibound state (Figure 4) can be found from the equation

$$
\left[K_{x}-j_{n}(x, E)\right] \phi_{n t}(x)=\lambda_{n t} \phi_{n t}(x)
$$

where the energy is counted from the top of the bottleneck potential, $\tilde{U}_{n, \max }$, and $\lambda_{n t}$ is negative. The transmission coefficient of a barrier with the quasibound state was calculated in Ref. 9], and is given by

$$
T_{n t}\left(E-E_{n}\right)=t_{n}\left(E-E_{n}\right)+\frac{m}{\imath k \hbar^{2}} \frac{\left\langle\phi_{n t}\left|j_{n}(x, E)\right| \chi_{n k}^{+}\right\rangle\left\langle\phi_{n t}\left|j_{n}(x, E)\right| \chi_{n k}^{-}\right\rangle}{E-E_{n}-\tilde{U}_{n, \max }-\bar{\lambda}_{n t}+\imath \Gamma_{n t}},
$$

where $\bar{\lambda}_{n t}=\lambda_{n t}+\delta \lambda_{n t}$ (with $\delta \lambda_{n t}$ accounting for the energy shift due to the possibility of tunneling in and out of the quasibound state) and the width of the tunneling resonance, $\Gamma_{n t}$, has the form $[9]$

$$
\Gamma_{n t}=\frac{m}{2 k \hbar^{2}}\left(\left|\left\langle\phi_{n t}\left|j_{n}(x, E)\right| \chi_{n k}^{+}\right\rangle\right|^{2}+\left|\left\langle\phi_{n t}\left|j_{n}(x, E)\right| \chi_{n k}^{-}\right\rangle\right|^{2}\right) .
$$

Substituting the expressions for the exchange term, Eq. (40), into Eq. (42), we obtain

$$
T_{n t}\left(E-E_{n}\right)=t_{n}\left(E-E_{n}\right)+\frac{K_{n}\left[T_{0 t}\left(E-E_{0}\right)-T_{0 s}\left(E-E_{0}\right)\right]^{2}}{E-E_{n}-\tilde{U}_{n, \max }-\bar{\lambda}_{n t}+\imath \Gamma_{n t}\left(E-E_{0}\right)},
$$

where $K_{n}$ is a scalar coefficient. The bottleneck potential of the fixed wire can also be assumed to be inverse parabolic, $\tilde{U}_{n}(x) \approx \tilde{U}_{n, \max }-m \Omega_{n}^{2} x^{2} / 2$, and the background transmission coefficient has the form

$$
t_{n}\left(E-E_{n}\right)=t\left[\frac{E-E_{n}-\tilde{U}_{n, \max }}{\hbar \Omega_{n}}\right] .
$$

The absolute value of the transmission coefficient Eq. (44) should not exceed unity. One can see that this condition is obeyed because the two terms in this expression are non-zero for different energies. 
For the singlet state there is no dip in the potential, but the barrier is a little bit higher than $\tilde{U}_{n, \max }$, which can be taken into account by introducing parameter $\delta j_{n}\left(E-E_{0}\right)$ proportional to $\left[T_{0 t}\left(E-E_{0}\right)-T_{0 s}\left(E-E_{0}\right)\right] \delta \tilde{j}_{n}$, so that the transmission coefficient for the singlet state can be written as

$$
T_{n s}\left(E-E_{n}\right)=t\left[\frac{E-E_{n}-\tilde{U}_{n, \max }-\delta j_{n}\left(E-E_{0}\right)}{\hbar \Omega_{n}}\right] .
$$

Finally, the width of the tunneling resonance takes form

$$
\Gamma_{n t}\left(E-E_{0}\right)=\Gamma_{n, 0}\left[T_{0 t}\left(E-E_{0}\right)-T_{0 s}\left(E-E_{0}\right)\right]^{2},
$$

where $\Gamma_{n, 0}$ is a constant.

2. Antiferromagnetic coupling $\left(j_{n}(x)<0\right)$.

In this case the singlet state experiences scattering through quazibonding state, whose bare energy and zero-order wave function can be determined by the equation

$$
\left[K_{x}+3 j_{n}(x, E)\right] \phi_{n s}^{\prime}(x)=\lambda_{n s}^{\prime} \phi_{n s}^{\prime}(x)
$$

Employing the same procedure as in the previous case, we obtain the transmission coefficients as

$$
\begin{gathered}
T_{n s}^{\prime}\left(E-E_{n}\right)=t_{n}\left(E-E_{n}\right)+\frac{K_{n}^{\prime}\left[T_{0 t}\left(E-E_{0}\right)-T_{0 s}\left(E-E_{0}\right)\right]^{2}}{E-E_{n}-\tilde{U}_{n, \max }-\bar{\lambda}_{n s}^{\prime}+\imath \Gamma_{n s}^{\prime}\left(E-E_{0}\right)}, \\
T_{n t}^{\prime}\left(E-E_{n}\right)=t\left[\frac{E-E_{n}-\tilde{U}_{n, \max }-\delta j_{n}^{\prime}\left(E-E_{0}\right)}{\hbar \Omega_{n}}\right] .
\end{gathered}
$$

In these expressions

$$
\Gamma_{n s}^{\prime}\left(E-E_{0}\right)=\Gamma_{n, 0}^{\prime}\left[T_{0 t}\left(E-E_{0}\right)-T_{0 s}\left(E-E_{0}\right)\right]^{2}
$$

We can establish approximate relations between the coefficients in the ferromagnetic and antiferromagnetic cases: $K_{n}^{\prime} \approx 9 K_{n}, \Gamma_{n}^{\prime} \approx 9 \Gamma_{n}$, and $\delta j_{n}^{\prime} \approx 3 \delta j_{n}$.

With these transmission coefficients we can obtain an expression for the conductance as

$$
G_{F W}=\frac{2 e^{2}}{h} \sum_{n}\left[\frac{3}{4}\left|T_{n t}\left(E-E_{n}\right)\right|^{2}+\frac{1}{4}\left|T_{n s}\left(E-E_{n}\right)\right|^{2}\right] .
$$

The conductances of the swept and fixed wires is shown in Figure 5 as functions of the gate voltage of the swept wire (which determines the energy separation of the local state, $E_{0}$, and the Fermi energy) for the ferromagnetic case and for the following set of parameters: $E_{F}-$ 
$\tilde{U}_{\max }=0.6 \mathrm{meV}, \tilde{U}_{\max }-E_{n}=0.3 \mathrm{meV}, J_{\max }=0.3 \mathrm{meV}, \omega_{-}=0.3 \mathrm{meV}, \omega_{+}=1.5 \mathrm{meV}, \omega_{U}=$ $1 \mathrm{meV}, \Omega_{n}=1 \mathrm{meV}, K_{n}=0.0285 \mathrm{meV}, \Gamma_{n}=0.1 \mathrm{meV}$, and $\delta j_{n}=0.1 \mathrm{meV}$. The confinement potential in the fixed wire is assumed to be parabolic with the level separation $E_{n}-E_{n-1}=$ $0.3 \mathrm{meV}$.

One can see from this figure that the conductance peak in the fixed wire appears exactly at the same gate voltages as the 0.75 -plateau in the conductance of the swept wire indicating their common nature as the local moment formation as the swept wire pinches off.

\section{CONCLUSIONS}

In this report, we have presented a comprehensive theory for the electron dynamics in a system of coupled quantum wires, under conditions where a local magnetic moment is formed in one of them. Rather than assume that this local moment is related to the formation of an associated localized state in the swept wire, we have calculated the single-electron transmission properties of the fixed wire in a potential that is modified by the presence of

an extra scattering term, arising from the presence of the local moment in the swept wire. To determine the transmission coefficients in this system, we derived equations describing the dynamics of electrons in the swept and fixed wires of the coupled-wire geometry. Our analysis clearly shows that the observation of a resonant peak in the conductance of the fixed wire is correlated to the appearance of additional structure (near $0.75 \cdot$ or $0.25 \cdot 2 e^{2} / h$ ) in the conductance of the swept wire, in agreement with the experimental results of Ref. [6].

\section{Acknowledgement}

The authors would like to thank S. A. Gurvitz, A. Yu. Smirnov and I. M. Djuric for the useful discussion and critical comments.

\section{APPENDIX A: ELECTRON SCATTERING BY A LOCALIZED SPIN}

In this appendix we discuss the canonical transformation from the uncoupled representation to the coupled representation.

The basis vectors in the spin space of electron spin and the magnetic moment in the uncoupled representation are given by Eqs. (3i). The form of the exchange operator $\vec{\sigma} \cdot \vec{S}$ in 
this basis is

$$
\hat{Q}=\vec{\sigma} \cdot \vec{S}=\left(\begin{array}{cccc}
1 & 0 & 0 & 0 \\
0 & 1 & 0 & 0 \\
0 & 0 & -1 & 2 \\
0 & 0 & 2 & -1
\end{array}\right)
$$

This operator can be diagonalized by a canonical transformation

$$
\hat{Q}^{\prime}=\hat{X}^{+} \hat{Q} \hat{X}=\left(\begin{array}{cccc}
1 & 0 & 0 & 0 \\
0 & 1 & 0 & 0 \\
0 & 0 & 1 & 0 \\
0 & 0 & 0 & -3
\end{array}\right)
$$

where the transformation operator is given by

$$
\hat{X}=\left(\begin{array}{cccc}
1 & 0 & 0 & 0 \\
0 & 1 & 0 & 0 \\
0 & 0 & \frac{1}{\sqrt{2}} & -\frac{1}{\sqrt{2}} \\
0 & 0 & \frac{1}{\sqrt{2}} & \frac{1}{\sqrt{2}}
\end{array}\right)
$$

The wave function is transformed in a similar way:

$$
\hat{\varphi}^{\prime}(x)=\hat{X}^{+} \hat{\varphi}(x)
$$

The equation describing scattering of an electron on LMM is

$$
\left(\epsilon-K_{x}-U(x)+J(x) \vec{\sigma} \cdot \vec{S}\right) \varphi(x)=0,
$$

where $\varphi(x)$ is a four-component wave function in spin space:

$$
\hat{\varphi}(x)=\sum_{\alpha=1}^{4} \chi_{\alpha} \varphi_{\alpha}(x) .
$$

This equation can be formally solved with help of the canonical transformation, Eq. (A3) $\left(\hat{I}=\hat{X}^{+} \hat{X}\right)$ :

$$
\begin{array}{r}
\hat{X}^{+}\left(\epsilon-K_{x}-U(x)+J(x) \vec{\sigma} \cdot \vec{S}\right) \hat{X} \hat{X}^{+} \varphi(x) \\
=\left(\epsilon-K_{x}-U(x)+J(x) \hat{X}^{+} \vec{\sigma} \cdot \vec{S} \hat{X}\right) \hat{\varphi}^{\prime}(x) \\
\quad=\left(\epsilon-K_{x}-U(x)+J(x) \hat{Q}^{\prime}\right) \hat{\varphi}^{\prime}(x)=0 .
\end{array}
$$


Equation (A7) is diagonal in spin space and can be written as four equations for wave function components:

$$
\begin{gathered}
\left(\epsilon-K_{x}-U(x)+J(x)\right) \varphi_{\alpha}^{\prime}(x)=0, \alpha=1,2,3 \\
\left(\epsilon-K_{x}-U(x)-3 J(x)\right) \varphi_{4}^{\prime}(x)=0 .
\end{gathered}
$$

\section{APPENDIX B: GREEN'S FUNCTION FOR AN ELECTRON SCATTERED BY} A LOCALIZED SPIN

In this Appendix we derive the Green's function of Eq. (20), starting from its definition, Eq. (14),

$$
\hat{G}_{0}(\epsilon)=\left[\epsilon-K_{x}-U_{0}(x)+J(x) \hat{\vec{\sigma}} \cdot \hat{\vec{S}}\right]^{-1}
$$

According to this, the Green's function satisfies the equation

$$
\left(\epsilon-K_{x}-U(x)+J(x) \hat{\vec{\sigma}} \cdot \hat{\vec{S}}\right) \hat{G}\left(x, x^{\prime}, \epsilon\right)=\hat{I} \delta\left(x-x^{\prime}\right),
$$

where $\hat{I}$ is the unit matrix in spin space, together with the boundary conditions, which depend on the particular kind of the Green's function that we are looking for.

Using the canonical transformation of Appendix we can calculate the Green's function $\hat{G}^{\prime}\left(x, x^{\prime}, \epsilon\right)=\hat{X}^{+} \hat{G}\left(x, x^{\prime}, \epsilon\right) \hat{X}$, which is diagonal in spin space, $G_{\alpha \beta}^{\prime}\left(x, x^{\prime}, \epsilon\right)=\delta_{\alpha \beta} G_{\alpha}^{\prime}\left(x, x^{\prime}, \epsilon\right)$, and whose components, $G_{\alpha}^{\prime}\left(x, x^{\prime}, \epsilon\right)=g^{t}\left(x, x^{\prime}, \epsilon\right)$ for $\alpha=1,2,3$, and $G_{4}^{\prime}\left(x, x^{\prime}, \epsilon\right)=$ $g^{s}\left(x, x^{\prime}, \epsilon\right)$, should satisfy the equations

$$
\begin{gathered}
\left(\epsilon-K_{x}-U(x)+J(x)\right) g^{t}\left(x, x^{\prime}, \epsilon\right)=\delta\left(x-x^{\prime}\right) \\
\left(\epsilon-K_{x}-U(x)-3 J(x)\right) g^{s}\left(x, x^{\prime}, \epsilon\right)=\delta\left(x-x^{\prime}\right) .
\end{gathered}
$$

The outgoing-wave Green's function, $G_{\alpha \beta}\left(x, x^{\prime}, \epsilon\right) \sim \delta_{\alpha, \beta} e^{ \pm i k x}$ for $x \longrightarrow \pm \infty$, is of most interest to us. It is shown in Ref. 9] that in terms of the scattering solutions the components of the desired Green's function are given by

$$
g^{t, s}\left(x, x^{\prime}, \epsilon\right)=\frac{m}{i k T_{t, s}}\left\{\begin{array}{l}
\phi_{k}^{t, s-}\left(x^{\prime}\right) \phi_{k}^{t, s+}(x) \text { if } x>x^{\prime}, \\
\phi_{k}^{t, s+}\left(x^{\prime}\right) \phi_{k}^{t, s-}(x) \text { if } x<x^{\prime}
\end{array}\right.
$$


where $k=\sqrt{2 m \epsilon} / \hbar$ and $\phi_{k}^{t, s-/+}$ are triplet/singlet scattering solutions originated from $+/-$ $\infty$, respectively.

Now the Green's function $\hat{G}^{\prime}\left(x, x^{\prime}, \epsilon\right)$ takes form

$$
\hat{G}^{\prime}\left(x, x^{\prime}, \epsilon\right)=\left(\begin{array}{cccc}
g^{t}\left(x, x^{\prime}, \epsilon\right) & 0 & 0 & 0 \\
0 & g^{t}\left(x, x^{\prime}, \epsilon\right) & 0 & 0 \\
0 & 0 & g^{t}\left(x, x^{\prime}, \epsilon\right) & 0 \\
0 & 0 & 0 & g^{s}\left(x, x^{\prime}, \epsilon\right)
\end{array}\right)
$$

and applying the canonical transformation backwards we obtain

$$
\left(\begin{array}{cccc}
g^{t}\left(x, x^{\prime}, \epsilon\right) & 0 & 0 & \hat{G}\left(x, x^{\prime}, \epsilon\right)=\hat{X} \hat{G}^{\prime}\left(x, x^{\prime}, \epsilon\right) \hat{X}^{+}= \\
0 & g^{t}\left(x, x^{\prime}, \epsilon\right) & 0 & 0 \\
0 & 0 & \frac{1}{2}\left[g^{t}\left(x, x^{\prime}, \epsilon\right)+g^{s}\left(x, x^{\prime}, \epsilon\right)\right] & \frac{1}{2}\left[g^{t}\left(x, x^{\prime}, \epsilon\right)-g^{s}\left(x, x^{\prime}, \epsilon\right)\right] \\
0 & 0 & \frac{1}{2}\left[g^{t}\left(x, x^{\prime}, \epsilon\right)-g^{s}\left(x, x^{\prime}, \epsilon\right)\right] & \frac{1}{2}\left[g^{t}\left(x, x^{\prime}, \epsilon\right)+g^{s}\left(x, x^{\prime}, \epsilon\right)\right]
\end{array}\right)
$$

Finally, Eq. (B7) can be split into the scalar part, proportional to a unit matrix in the spin space, and the part proportional to the exchange operator, $\hat{Q}=\hat{\vec{\sigma}} \cdot \hat{\vec{S}}$ :

$$
\hat{G}\left(x, x^{\prime}, \epsilon\right)=\frac{1}{4}\left[3 g^{t}\left(x, x^{\prime}, \epsilon\right)+g^{s}\left(x, x^{\prime}, \epsilon\right)\right] \hat{I}+\frac{1}{4}\left[g^{t}\left(x, x^{\prime}, \epsilon\right)-g^{s}\left(x, x^{\prime}, \epsilon\right)\right] \hat{\vec{\sigma}} \cdot \hat{\vec{S}}
$$

\section{APPENDIX C: GREEN'S FUNCTIONS AND TRANSMISSION COEFFI- CIENTS OF AN INVERSE PARABOLIC BARRIER}

In this section we briefly summarize some known facts about an inverse parabolic barrier 14]. If the barrier potential is given by $u(x)=-m \omega^{2} x^{2} / 2$, the scattering solutions of the equation

$$
\left[\epsilon-K_{x}-u(x)\right] \Psi^{ \pm}(x)=0
$$

are given by

$$
\Psi^{ \pm}(x)=\mathrm{E}(\eta, \pm \xi)
$$

where $\mathrm{E}(\eta, \xi)$ is a Weber function, i.e. a solution of the equation for parabolic cylinder functions, $y^{\prime \prime}(\xi)+\left(\frac{1}{4} \xi^{2}-\eta\right) y(\xi)=0,[16] ; \xi=q x$, and $q=\sqrt{2 m \omega / \hbar}$, whereas $\eta=-\epsilon /(\hbar \omega)$. 
The one-dimensional Green's function for such a barrier is given by

$$
G\left(x, x^{\prime}, \epsilon\right)=\frac{m t(\eta)}{\hbar^{2} q}\left\{\begin{array}{l}
\mathrm{E}(\eta, \xi) \mathrm{E}\left(\eta,-\xi^{\prime}\right), x>x^{\prime} \\
\mathrm{E}(\eta,-\xi) \mathrm{E}\left(\eta, \xi^{\prime}\right), x<x^{\prime}
\end{array},\right.
$$

where the transmission coefficient has the form

$$
t(\eta)=\left[1+e^{-2 \pi \eta}\right]^{-1 / 2}
$$

[1] S. Datta, Electron Transport in Mesoscopic Systems, Cambridge University Press, UK (1995).

[2] J. P. Bird and Y. Ochiai, Science 303, 1621 (2004).

[3] Y. Meir, K. Hirose, and N. S. Wingreen, Phys. Rev. Lett. 89, 196802 (2002).

[4] K.-F. Berggren and I. I. Yakimenko, Phys. Rev. B 66, 085323 (2002).

[5] K. Hirose, Y. Meir, and N. S. Wingreen, Phys. Rev. Lett. 90, 026804 (2003).

[6] T. Morimoto, Y. Iwase, N. Aoki, T. Sasaki, Y. Ochiai, A. Shailos, J. P. Bird, M. P. Lilly, J. L. Reno, and J. A. Simmons, Appl. Phys. Lett. 82, 3952 (2003).

[7] V. I. Puller, L. G. Mourokh, A. Shailos, and J. P. Bird, Phys. Rev. Lett. 92, 96802 (2004).

[8] V. I. Puller, L. G. Mourokh, A. Shailos, and J. P. Bird, IEEE Trans. on Nanotech., in press; cond-mat.

[9] S. A. Gurvitz and Y. B. Levinson, Phys. Rev. B 47, 10578 (1993).

[10] J. U. Nöckel and A. D. Stone, Phys. Rev. B 50, 17415 (1994).

[11] T. Rejec, A. Ramšak, and J. H. Jefferson, Phys. Rev. B 62, 12985 (2000).

[12] V. V. Flambaum and M. Yu. Kuchiev, Phys. Rev. B 61, R7869 (2000).

[13] Ch. Kunze and Ph. Bagwell, Phys. Rev. B 51, 13410 (1995).

[14] see e.g., Y. B. Levinson, M. I. Lubin, and E. V. Sukhorukov, Phys. Rev. B 45, 11936 (1992).

[15] J. P. Bird, et al., to be published.

[16] Handbook of Mathematical Functions, edited by M. Abramowitz and A. Stegun (National Bereau of Standards, Washington, D.C., 1964). 

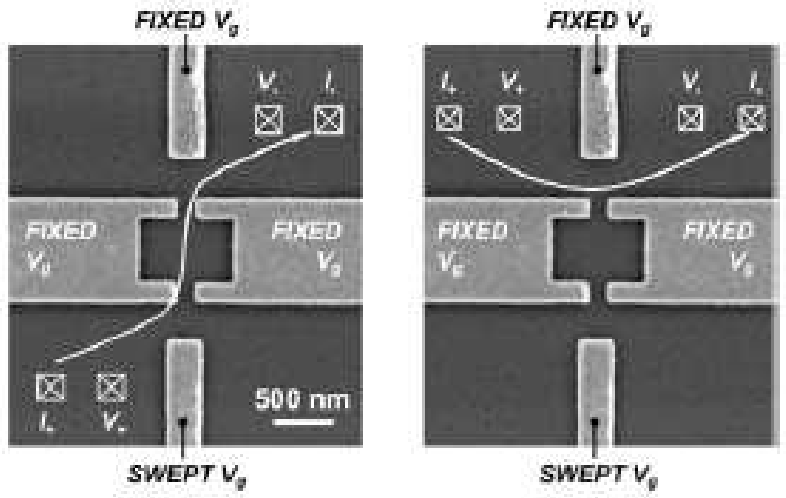

V. I. Puller, et al., Figure 1 of 5 

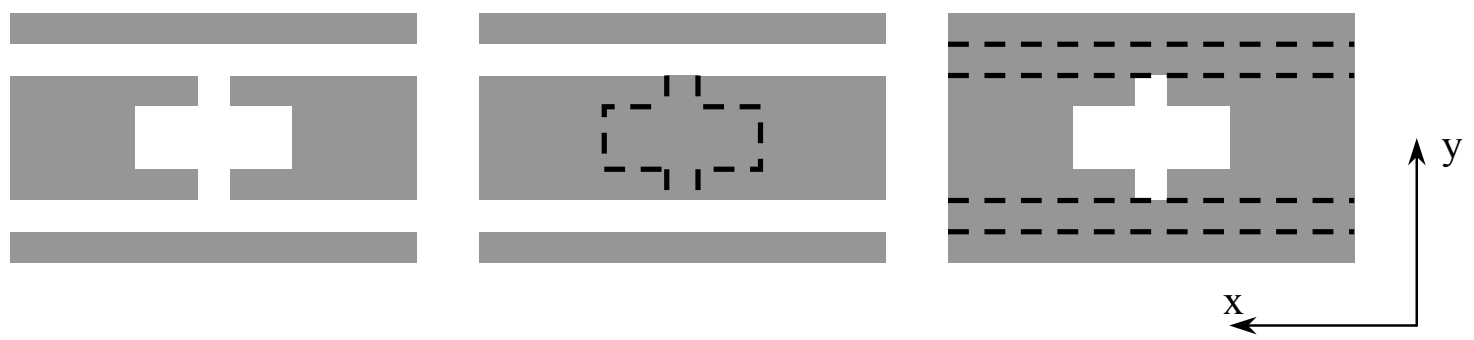

V. I. Puller, et al., Figure 2 of 5. 


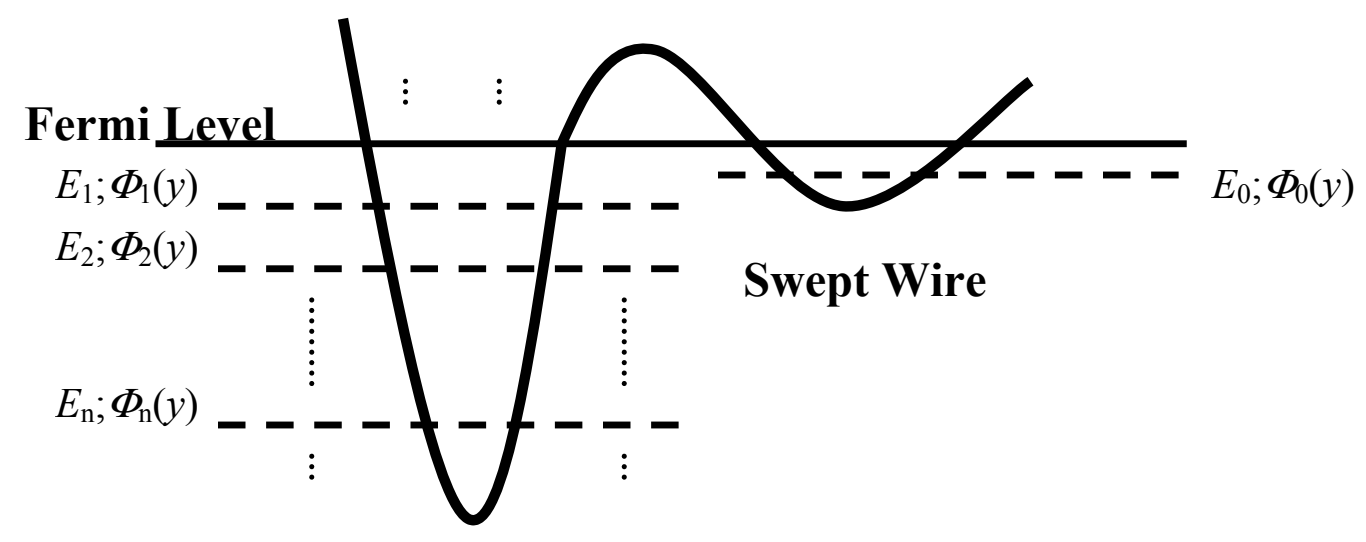

Fixed Wire

V. I. Puller, et al., Figure 3 of 5 


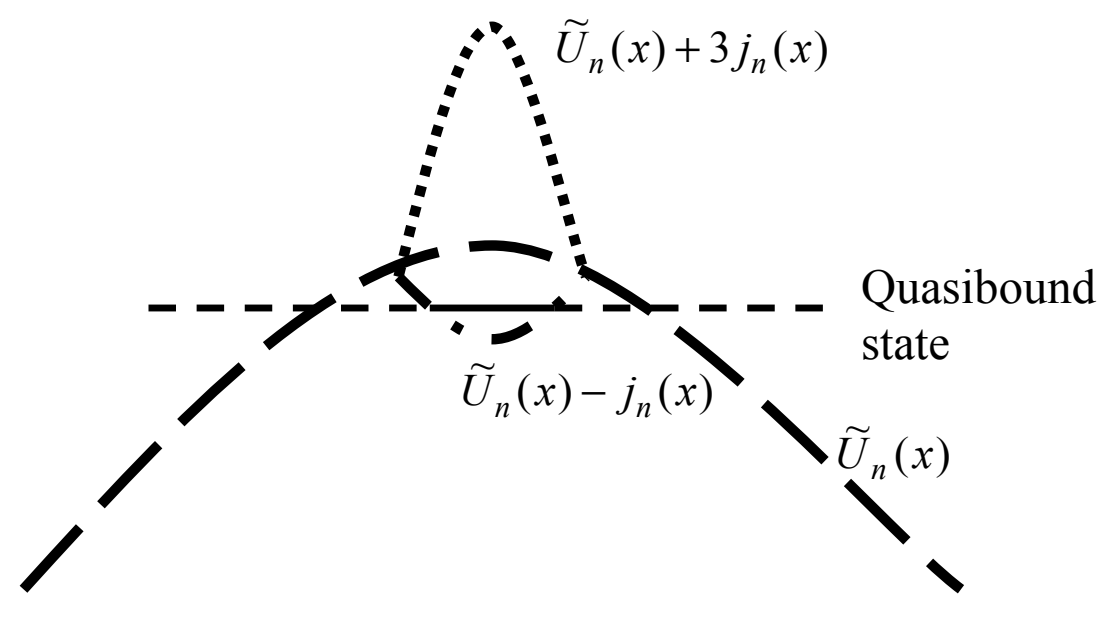

V. I. Puller, et al., Figure 4 of 5 


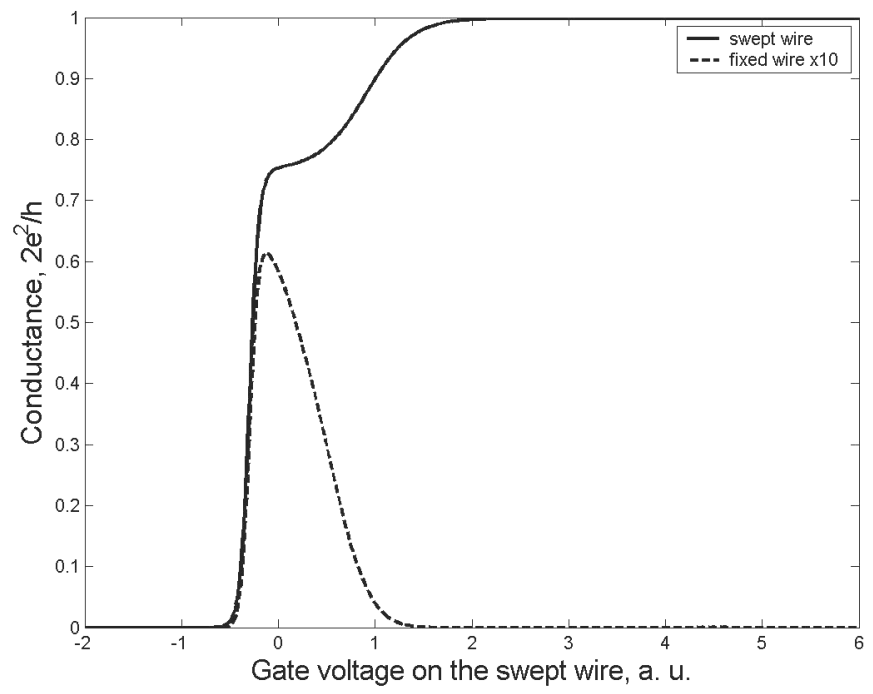

V. I. Puller, et al., Figure 5 of 5 